\title{
The Duration of Breastfeeding and Its Association with Metabolic Syndrome among Obese Children
}

\author{
Renata Yakubov, ${ }^{1}$ Erez Nadir, ${ }^{2}$ Roni Stein, ${ }^{1}$ and Adi Klein-Kremer ${ }^{1}$ \\ ${ }^{1}$ Department of Pediatrics, Hillel Yaffe Medical Center, 38100 Hadera, Israel \\ ${ }^{2}$ Department of Neonatology, Hillel Yaffe Medical Center, 38100 Hadera, Israel \\ Correspondence should be addressed to Erez Nadir; erezn@hy.health.gov.il
}

Received 11 May 2015; Accepted 22 July 2015

Academic Editor: Naval Vikram

Copyright (c) 2015 Renata Yakubov et al. This is an open access article distributed under the Creative Commons Attribution License, which permits unrestricted use, distribution, and reproduction in any medium, provided the original work is properly cited.

\begin{abstract}
Objective. The objective of this study was to evaluate whether duration of breastfeeding is associated with a lower prevalence of metabolic syndrome in obese children. Methods. A retrospective analysis of obese children aged 3 to 18 years followed at a pediatric outpatient clinic at a single center between the years 2008 and 2012. The children were divided according to their breastfeeding duration: no breastfeeding, a short period of breastfeeding, and a long term breastfeeding. Also, they were divided into metabolic and nonmetabolic syndrome groups, based on physical examination and laboratory tests. Results. Out of 4642 children who visited the clinic, 123 were obese and were included in the study. About half of them matched the metabolic syndrome criteria. There was no correlation between the prevalence of metabolic syndrome and the duration of breastfeeding. Hypertension, abnormal low levels of HDL, high levels of HbAlc, and high fasting triglyceride levels were very common in our study population, yet no statistical significance was noted among the different breastfeeding groups. Conclusion. In this study, breastfeeding was not associated with a reduced risk for metabolic syndrome, compared with formula feeding, in children who are obese.
\end{abstract}

\section{Introduction}

Over the past decades, the prevalence of childhood obesity has grown worldwide [1-5]. Therapeutic interventions aimed at encouraging weight loss in obese children are costly and have had unsatisfactory long-term success rates [6]. Therefore, the identification of programs and strategies to prevent obesity more effectively has drawn much attention in recent years. Simple and cheap strategies without potential side effects are preferable. The concept that nutrition in infancy may have a long-term influence on the development of obesity first emerged in the 1960s with the pioneering work of McCance [7]. Recently, systematic reviews [8-11], based on population studies, have confirmed an association between breastfeeding and lower risk for developing obesity in adulthood. Owen et al. [12] reviewed studies which investigated the relation between breastfeeding and total cholesterol and low-density lipoprotein (LDL) cholesterol. One of their conclusions was that in adulthood, breastfed subjects had lower levels of total cholesterol than bottlefed subjects. Another meta-analysis examined the relation between breastfeeding and blood pressure [13]. Mean systolic blood pressure was found to be lower at adulthood in breastfed participants than in bottle-fed ones. Another study [14] examined blood pressure in 216 adolescents who were born preterm and observed that those who were fed breast milk had a lower mean arterial blood pressure than those who were fed preterm formula. Regarding developing diabetes mellitus type 2 in adulthood, a systematic review [15] showed that breastfeeding, compared to formula-feeding, was associated with a lower risk for type 2 diabetes mellitus by almost $40 \%$. Furthermore, it had a positive effect on fasting glucose and insulin levels. The association between breastfeeding and childhood obesity is uncertain, and results are inconsistent: some studies show a strong association between breastfeeding and childhood obesity $[11,13,14]$ while others do not [1618]. There are clinical studies that show that breastfeeding decreases the risk for adulthood obesity [8-11]. 
We have found no published studies testing the influence of the duration of breastfeeding on different aspects of the metabolic syndrome.

\section{Materials and Methods}

2.1. Design. In this study, we examined the association between breastfeeding duration and metabolic syndrome. Metabolic syndrome was defined by the presence of 3 out of 5 criteria: BMI $\geq 95$ th percentile for age and sex, hypertriglyceridemia (>120 mg/dL), high blood pressure ( $>95$ th percentile for age, sex, and height), high blood fasting glucose level (>100 mg/dL), and low HDL level $(<45 \mathrm{mg} / \mathrm{dL})$ [19]. In addition we examined the relationship between the breastfeeding duration and different laboratory tests (such as fasting blood cholesterol level, fasting blood triglycerides level, HbAlc, glucose, and insulin levels) and the systolic and diastolic blood pressures.

2.2. Patients' Selection. In this retrospective analysis, we included children aged 3-18 years, who visited the pediatric or pediatric nephrology outpatient clinics of a single center (Hillel Yaffe Medical Center, Harera, Israel) for hypertension and/or obesity (BMI > 95th percentile for age and sex), with or without metabolic syndrome, in the years 2008 to 2012.

2.3. Breastfeeding History. Duration of breastfeeding was noted and divided into three groups:

Group 1: none (0-2 months),

Group 2: short period (2-6 months),

Group 3: long term (6 months and above) breastfeeding.

2.4. Physical Measurements. Blood pressure (BP) was measured using the oscillometric method. Hypertension (HTN) was defined as either systolic and/or diastolic blood pressure $\geq 95$ th percentile measured on three or more occasions. The degree of HTN is further delineated by the two stages [20]:

Stage 1 hypertension: systolic and/or diastolic BP among the 95th percentile and $5 \mathrm{mmHg}$ above the 99th percentile, or if in adolescents the BP exceeds $140 / 90 \mathrm{mmHg}$ even when $<95$ th percentile.

Stage 2 hypertension: systolic and/or diastolic BP $\geq 99$ th percentile plus $5 \mathrm{mmHg}$.

Height and weight were measured using standardized procedures. Obesity was defined as body mass index (BMI) above $95 \%$ percentile for age and sex according to Centers for Disease Control and Prevention (CDC) chart [21].

2.5. Demographic and Ethnic Background. The children included (123) were of Jewish or Arab descents. All live around "Hadera" district in Israel.

2.6. Laboratory Assays and Methods. Laboratory tests were performed in the hospital laboratory. 12-hour fasting blood sampling was collected and tested for glucose, total cholesterol, LDL, HDL, triglycerides, insulin, and HbAlc levels.
TABLE 1: Breastfeeding groups distributions of children with and without metabolic syndrome.

\begin{tabular}{lccc}
\hline & All Patients & $\begin{array}{c}\text { Metabolic } \\
\text { syndrome }\end{array}$ & $\begin{array}{c}\text { Non-metabolic } \\
\text { syndrome }\end{array}$ \\
\hline All & 123 & $58(47 \%)$ & $65(53 \%)$ \\
Group 1 & $57(46.5 \%)$ & $32(56 \%)$ & $25(44 \%)$ \\
Group 2 & $37(30 \%)$ & $13(35 \%)$ & $24(65 \%)$ \\
Group 3 & $29(23.5 \%)$ & $13(45 \%)$ & $16(55 \%)$ \\
$p$ & & 0.99 & 0.98 \\
\hline
\end{tabular}

Definition for deviation values for blood test was high HbAlc: above 5.8\%, impaired glucose tolerance: fasting glucose level above $100 \mathrm{mg} / \mathrm{dL}$, insulin resistance: insulin level above $180 \mathrm{pmol} / \mathrm{L}$, hypercholesterolemia: total cholesterol level above $200 \mathrm{mg} / \mathrm{dL}$, hyperlipidemia: LDL level above $130 \mathrm{mg} / \mathrm{dL}$, hypertriglyceridemia: TG level above $120 \mathrm{mg} / \mathrm{dL}$, and low HDL: level below $45 \mathrm{md} / \mathrm{dL}$ [22].

2.7. Exclusion Criteria. Either insufficient laboratory data, or a presence of a diseases and/or a medicinal treatment that could affect metabolic functions and/or patient's weight (for example diabetes mellitus, Cushing syndrome, thyroid disorders, febrile illness at time the blood sampling, and systemic steroid usage).

2.8. Statistical Analysis. We used Student's $t$-test for unpaired samples to compare parametric data and the $\chi^{2}$ test to compare nonparametric data between groups. Statistical significance was set at a $p$ value $<0.05$. All calculations were made using IBM SPSS software version 20 (International Business Machines Corp., New Orchard Road, Armonk, New York 10504). The study was approved by local board for human research. Informed consent was not needed as the study was retrospective, and collected data was unidentified.

\section{Results}

4642 children visited the pediatric or pediatric nephrology outpatient clinics during the study period. 168 (3.6\%) children had BMI above $95 \%$ and were included. Out of them, 33 failed to provide information regarding breastfeeding and 12 could not define whether they had metabolic syndrome: for 7 of them lipid profile results were unavailable, and for the other 5 information regarding blood pressure follow-up was missing. Hence the final sample was 123 children (59 males and 64 females; 57 Arabs and 66 Jews). There were no statistically significant differences regarding sex or ethnic origin among the breastfeeding groups.

Metabolic syndrome: about half $(47 \%)$ of the patients in this study matched the metabolic syndrome criteria [22]. These differences were not significant statistically in terms of the prevalence of metabolic syndrome among the subgroups (Table 1).

Table 2 summarizes the numbers and percents of children with hypertension, high $\mathrm{HbAlc}$, high glucose level, high LDL level, low HDL level, high TC level, high insulin level, and high TG level in the whole group of the included children 
TABLE 2: Count of children with various abnormal results among the different subgroups.

\begin{tabular}{lccccccccc}
\hline & All & HTN & HbAlc & Glucose & LDL & HDL & TC & Insulin & TG \\
\hline All & 123 & $60(49 \%)$ & $35(28.5 \%)$ & $13(10.5 \%)$ & $14(11 \%)$ & $80(65 \%)$ & $12(10 \%)$ & $22(18 \%)$ & $35(28.5 \%)$ \\
Group 1 & $57(46.5 \%)$ & $28(49 \%)$ & $16(28 \%)$ & $6(10.5 \%)$ & $4(7 \%)$ & $43(75 \%)$ & $5(9 \%)$ & $10(17.5 \%)$ & $23(40 \%)$ \\
Group 2 & $37(30 \%)$ & $19(51 \%)$ & $12(32 \%)$ & $4(10.5 \%)$ & $6(16 \%)$ & $18(48.5 \%)$ & $3(8 \%)$ & $7(19 \%)$ & $6(16 \%)$ \\
Group 3 & $29(23.5 \%)$ & $13(45 \%)$ & $7(24 \%)$ & $3(10.5 \%)$ & $4(14 \%)$ & $19(65.5 \%)$ & $4(14 \%)$ & $5(17 \%)$ & $6(20.5 \%)$ \\
$p$ & & 1.00 & 0.99 & 1.00 & 0.94 & 0.98 & 0.97 & 1.00 & 0.93 \\
\hline
\end{tabular}

and in the groups of breastfeeding. There was no statistically significant difference among the subgroups regarding any of the above variables.

\section{Discussion}

This study aimed to find a relation between the duration of breastfeeding and the occurrence of metabolic syndrome in the pediatric population. The prevalence of the metabolic syndrome was about half of the obese children. This finding is similar to that of a previous study [22] that also demonstrates a high risk of metabolic syndrome in obese children.

We did not find any correlation between the prevalence of metabolic syndrome and the duration of breastfeeding.

Many similar studies were published in the last years and, like ours, have not found a relationship between breastfeeding and obesity in childhood $[9,17]$. Nevertheless, some studies did find a correlation between breastfeeding duration and decreased incidence of metabolic syndrome later in life $[8,10,11]$. Thereupon, we can deduce that the matter is still controversial and that further investigation is needed regarding it.

In our study, we found no correlation between the duration of breastfeeding and abnormal concentrations of blood glucose and serum insulin.

There are only a few studies that tried to find a correlation between breastfeeding duration and type 2 diabetes mellitus. Furthermore, only in one study there was a positive correlation between breastfeeding duration and type 2 diabetes mellitus [15]. This subject is controversial, and further investigation is needed.

In our study, we found no significant difference among the breastfeeding groups regarding to the prevalence of hypertension. Searching medical literature, we found numerous studies regarding this issue, some of which have found reduced hypertension associated with breastfeeding [14, 23] and some have not [13].

Our study has several limitations; first, it is a small study in scope. In addition, it is a retrospective study. Moreover, patients' family history has not been taken into account and perhaps should have been.

\section{Conclusion}

In this study, breastfeeding was not associated with a reduced risk for metabolic syndrome, compared with formula feeding, in children who are obese.

\section{Abbreviations}

BMI: Body mass index

BP: $\quad$ Blood pressure

HbAlc: Hemoglobin Alc

HDL: High density lipoprotein

HTN: Hypertension

LDL: Low density lipoprotein

TC: Total cholesterol

TG: Triglycerides.

\section{Conflict of Interests}

The authors declare that there is no conflict of interests regarding the publication of this paper.

\section{References}

[1] C. Apfelbacher, J. Cairns, T. Bruckner et al., "Prevalence of overweight and obesity in East and West German children in the decade after reunification: population-based series of cross-sectional studies," Journal of Epidemiology \& Community Health, vol. 62, no. 2, pp. 125-130, 2008.

[2] M. E. G. Armstrong, M. I. Lambert, K. A. Sharwood, and E. V. Lambert, "Obesity and overweight in South African primary school children - the Health of the Nation study," South African Medical Journal, vol. 96, no. 5, pp. 439-444, 2006.

[3] L. J. Chen, K. R. Fox, A. Haase, and J. M. Wang, "Obesity, fitness and health in Taiwanese children and adolescents," European Journal of Clinical Nutrition, vol. 60, no. 12, pp. 1367-1375, 2006.

[4] K. M. Flegal and R. P. Troiano, "Changes in the distribution of body mass index of adults and children in the US population," International Journal of Obesity, vol. 24, no. 7, pp. 807-818, 2000.

[5] Y. Wang and T. Lobstein, "Worldwide trends in childhood overweight and obesity," International Journal of Pediatric Obesity, vol. 1, no. 1, pp. 11-25, 2006.

[6] "Periodic health examination, 1994 update: 1. Obesity in childhood. Canadian Task Force on the Periodic Health Examination," Canadian Medical Association Journal, vol. 150, no. 6, pp. 871-879, 1994.

[7] R. A. McCance, "Food, growth, and time," The Lancet, vol. 280, no. 7257, pp. 621-626, 1962.

[8] S. Arenz, R. Rückerl, B. Koletzko, and R. Von Kries, "Breastfeeding and childhood obesity-a systematic review," International Journal of Obesity, vol. 28, no. 10, pp. 1247-1256, 2004.

[9] C. G. Owen, R. M. Martin, P. H. Whincup, G. Davey-Smith, M. W. Gillman, and D. G. Cook, "The effect of breastfeeding on mean body mass index throughout life: a quantitative review of published and unpublished observational evidence," American Journal of Clinical Nutrition, vol. 82, no. 6, pp. 1298-1307, 2005. 
[10] C. G. Owen, R. M. Martin, P. H. Whincup, G. D. Smith, and D. G. Cook, "Effect of infant feeding on the risk of obesity across the life course: a quantitative review of published evidence," Pediatrics, vol. 115, no. 5, pp. 1367-1377, 2005.

[11] T. Harder, R. Bergmann, G. Kallischnigg, and A. Plagemann, "Duration of breastfeeding and risk of overweight: a metaanalysis," American Journal of Epidemiology, vol. 162, no. 5, pp. 397-403, 2005.

[12] C. G. Owen, P. H. Whincup, K. Odoki, J. A. Gilg, and D. G. Cook, "Infant feeding and blood cholesterol: a study in adolescents and a systematic review," Pediatrics, vol. 110, no. 3, pp. 597-608, 2002.

[13] C. G. Owen, P. H. Whincup, J. A. Gilg, and D. G. Cook, "Effect of breast feeding in infancy on blood pressure in later life: systematic review and meta-analysis," British Medical Journal, vol. 327, no. 7425, pp. 1189-1192, 2003.

[14] A. Singhal, T. J. Cole, and A. Lucas, "Early nutrition in preterm infants and later blood pressure: two cohorts after randomised trials," The Lancet, vol. 357, no. 9254, pp. 413-419, 2001.

[15] C. G. Owen, R. M. Martin, P. H. Whincup, G. D. Smith, and D. G. Cook, "Does breastfeeding influence risk of type 2 diabetes in later life? A quantitative analysis of published evidence," American Journal of Clinical Nutrition, vol. 84, no. 5, pp. 10431054, 2006.

[16] M. Vafa, N. Moslehi, S. Afshari, A. Hossini, and M. Eshraghian, "Relationship between breastfeeding and obesity in childhood," Journal of Health, Population and Nutrition, vol. 30, no. 3, pp. 303-310, 2012.

[17] M. Jiang and E. M. Foster, "Duration of breastfeeding and childhood obesity: a generalized propensity score approach," Health Services Research, vol. 48, no. 2, pp. 628-651, 2013.

[18] A. F. Haynos and W. T. O’Donohue, "Universal childhood and adolescent obesity prevention programs: review and critical analysis," Clinical Psychology Review, vol. 32, no. 5, pp. 383-399, 2012.

[19] R. Weiss, J. Dziura, T. S. Burgert et al., "Obesity and the metabolic syndrome in children and adolescents," The New England Journal of Medicine, vol. 350, no. 23, pp. 2362-2374, 2004.

[20] National High Blood Pressure Education Program Working Group on High Blood Pressure in C and Adolescents, "The fourth report on the diagnosis, evaluation, and treatment of high blood pressure in children and adolescents," Pediatrics, vol. 114, pp. 555-576, 2004.

[21] Centers for Disease Control and Prevention and National Center for Health Statistics, Clinical Growth Charts, Centers for Disease Control and Prevention, 2009, http://www.cdc.gov/growthcharts/clinical_charts.htm.

[22] S. R. Daniels and F. R. Greer, "Lipid screening and cardiovascular health in childhood," Pediatrics, vol. 122, no. 1, pp. 198-208, 2008.

[23] R. M. Martin, D. Gunnell, and G. D. Smith, "Breastfeeding in infancy and blood pressure in later life: systematic review and meta-analysis," American Journal of Epidemiology, vol. 161, no. 1, pp. 15-26, 2005. 


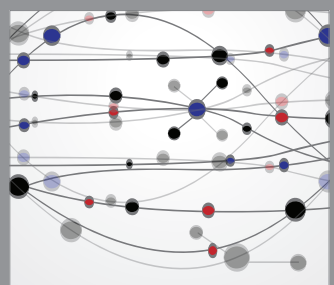

The Scientific World Journal
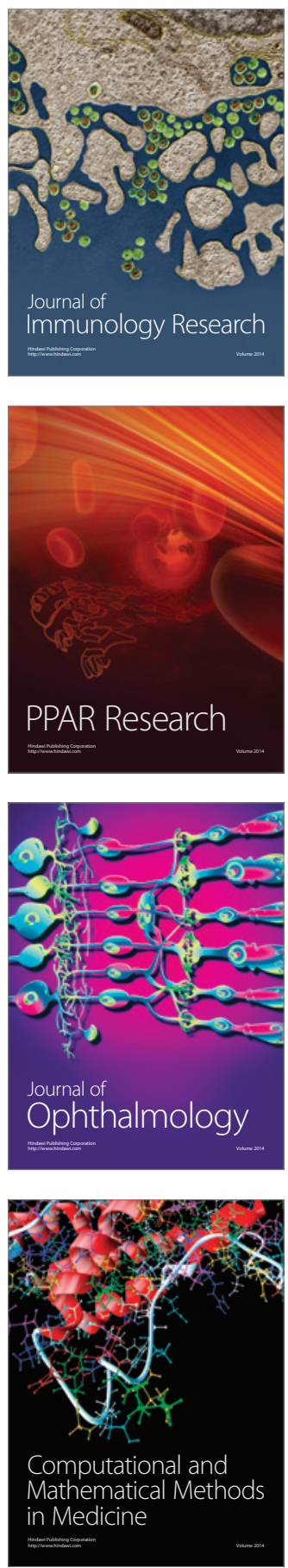

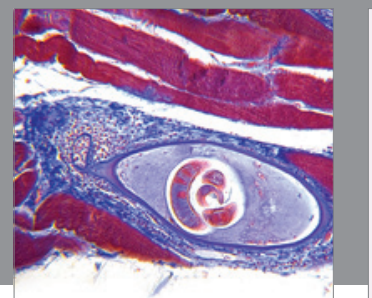

Gastroenterology

Research and Practice
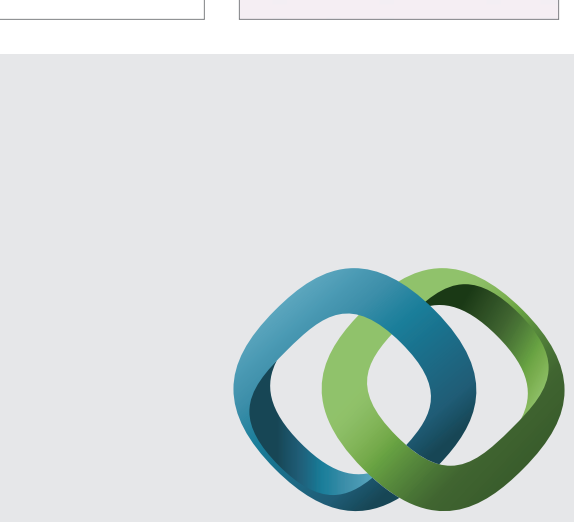

\section{Hindawi}

Submit your manuscripts at

http://www.hindawi.com
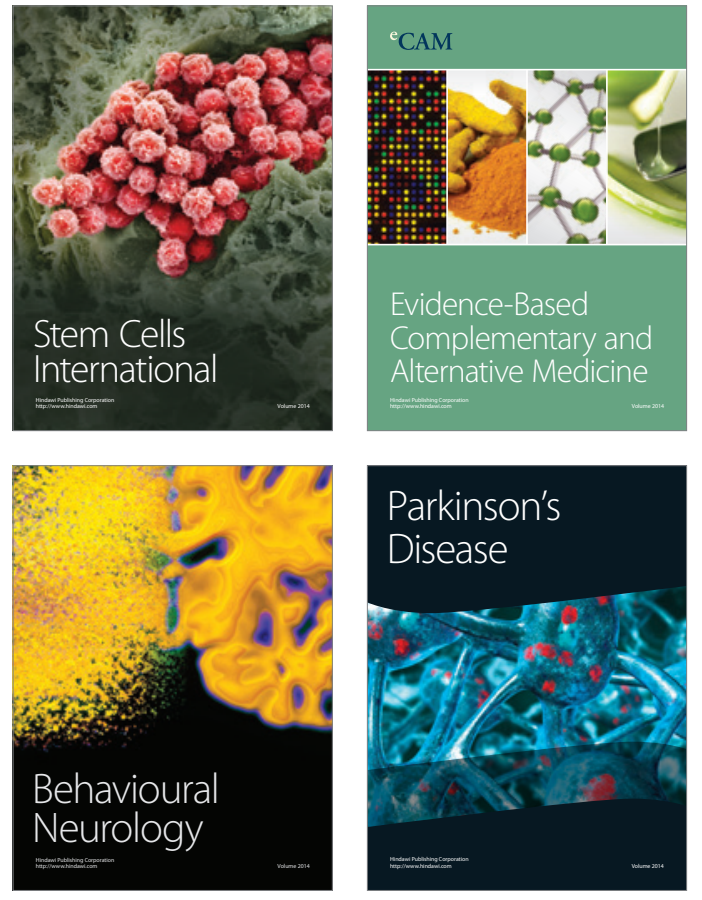
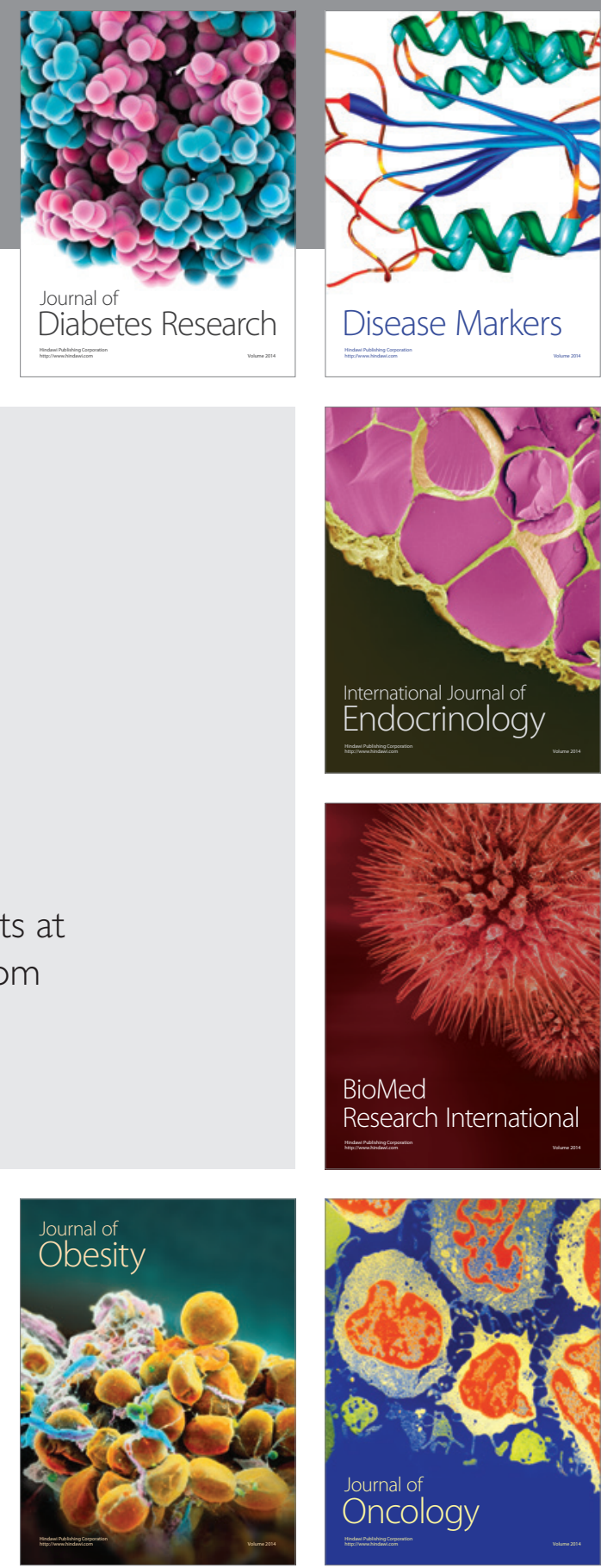

Disease Markers
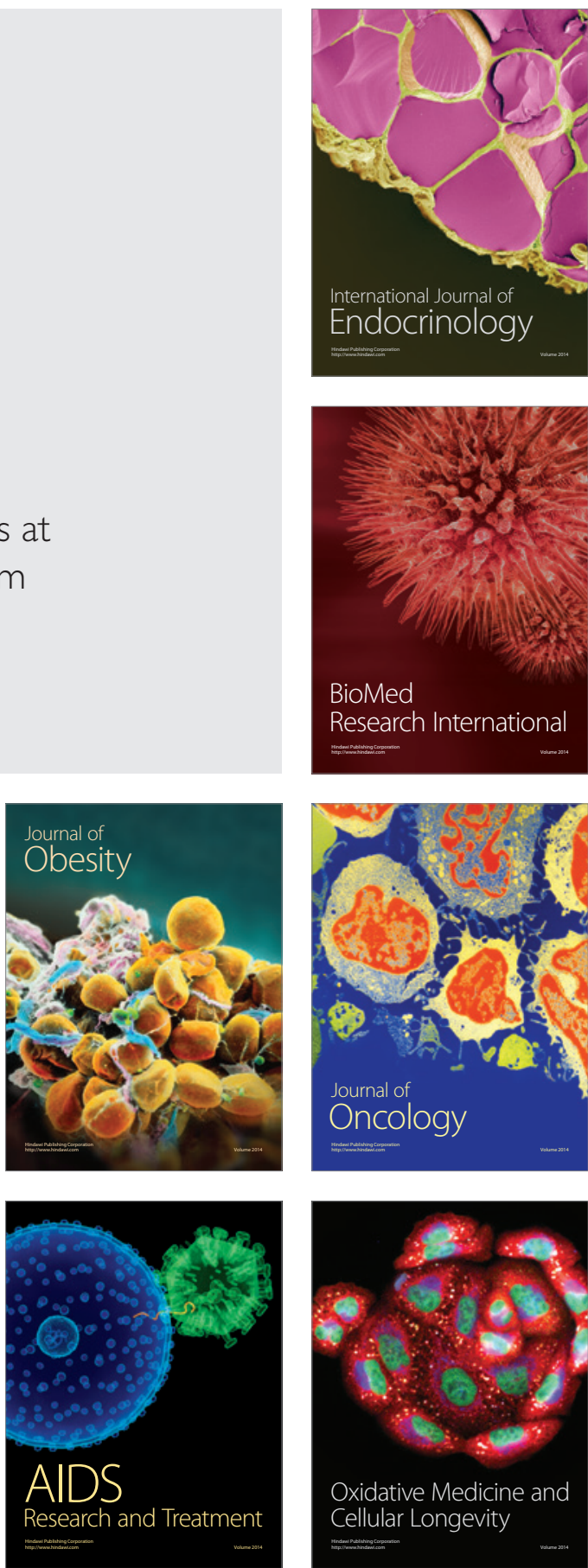